\title{
Numerical Simulations and Coherence Properties of Supercontinuum Generation in Photonic Crystal and Tapered Optical Fibers
}

\author{
John M. Dudley, Member, IEEE, and Stéphane Coen
}

\begin{abstract}
Numerical simulations have been used to study broad-band supercontinuum generation in optical fibers with dispersion and nonlinearity characteristics typical of photonic crystal or tapered fiber structures. The simulations include optical shock and Raman nonlinearity terms, with quantum noise taken into account phenomenologically by including in the input field a noise seed of one photon per mode with random phase. For input pulses of 150-fs duration injected in the anomalous dispersion regime, the effect of modulational instability is shown to lead to severe temporal jitter in the output, and associated fluctuations in the spectral amplitude and phase across the generated supercontinuum. The spectral phase fluctuations are quantified by performing multiple simulations and calculating both the standard deviation of the phase and, more rigorously, the degree of first-order coherence as a function of wavelength across the spectrum. By performing simulations over a range of input pulse durations and wavelengths, we can identify the conditions under which coherent supercontinua with a well-defined spectral phase are generated.
\end{abstract}

Index Terms-Nonlinear pulse propagation, optical fibers, photonic crystal fibers, supercontinuum generation, Raman solitons.

\section{INTRODUCTION}

$\mathbf{P}$ HOTONIC CRYSTAL FIBERS (PCF) commonly consist of a central-fused silica core surrounded by a regular array of air holes running along the fiber length. The number, size, and orientation of the air-holes around the core provide additional degrees of freedom not present in conventional fibers, and can be manipulated to yield novel confinement and dispersion characteristics. PCFs have thus been shown to exhibit several remarkable properties, including "endlessly single-mode" behavior [1], [2], novel group velocity dispersion (GVD) characteristics such as zero-dispersion wavelengths (ZDW) in the near-infrared [3]-[6], and enhanced effective nonlinearity due to the reduced mode size in the core [7]. With fiber structures designed so that the ZDW is shifted within the operating bandwidths of high-power near-infrared femtosecond lasers, these properties have been exploited to demonstrate

J. M. Dudley is with the Laboratoire d'Optique P. M. Duffieux, Université de Franche-Comté, 25030 Besançon, France (e-mail: john.dudley@univfcomte.fr).

S. Coen is with the Service d'Optique et Acoustique, Université Libre de Bruxelles, B-1050 Brussels, Belgium. soliton propagation effects around $850 \mathrm{~nm}$ [8], and the generation of broad-band supercontinuum (SC) spectra spanning over an octave [9], [10]. Such broad-band SC spectra have already found application in optical coherence tomography [11] and, particularly, in optical frequency metrology where they have allowed successful bridging of the optical-microwave frequency gap [12]-[14]. Similar broad-band SC have also been demonstrated in standard fiber tapered to a strand diameter of $\sim 2 \mu \mathrm{m}$ where the tapering introduces dispersion and nonlinearity characteristics similar to those of PCF [15], [16].

Numerical simulations of SC generation in PCF have also recently been reported, agreeing well with experimental observations and providing an improved understanding of the underlying spectral broadening mechanisms involved [17]-[19]. However, an aspect of SC generation in PCFs which has not yet been the subject of detailed study is the sensitivity of the spectral broadening processes to input pulse noise. This is nonetheless of particular importance, because key applications of octave-spanning SC sources such as precision optical frequency metrology require highly coherent SC light with limited pulse to pulse variations and reduced timing jitter [20]. In this regard, we note that $\mathrm{SC}$ generation in standard optical fiber has previously been the subject of much research in a telecommunications context, where spectrally sliced broad-band SC have been proposed as sources for wavelength-division-multiplexing applications [21]-[28]. Significantly, in this previous work, numerical simulations have shown that the generation of broad-band spectra using picosecond and subpicosecond pulse pumping in the presence of noise can, in fact, be associated with significant temporal jitter and a poorly defined spectral phase across the generated broad-band spectrum [23], [25], [26].

In this paper, we present results of numerical simulations of SC generation in PCF and tapered fibers which study, in particular, the fluctuations in both the temporal and spectral properties introduced by the presence of noise on the input pulse. The paper is organized as follows. In Section II, we describe our numerical model, and give the fiber parameters used in the simulations. In order to discuss the main physical mechanisms involved in SC generation, Section III.A presents results for input pulses injected in the fiber anomalous dispersion regime in the absence of noise. In Section III.B, the effect of input pulse noise is then discussed, and shown to be associated with fluctuations in the spectral phase across the SC. By quantifying the phase fluctuations using the mutual degree of coherence calculated over an ensemble of simulations, the influence of the input pulse wavelength and duration on the SC coherence is studied, allowing 
optimal experimental conditions for coherent SC generation to be identified. Section IV discusses several implications of these results and concludes the paper.

\section{NUMERICAL MODEL}

Our numerical model uses a generalized scalar propagation equation suitable for studying broad-band pulse evolution in optical fibers [18], [29]

$$
\begin{aligned}
\frac{\partial A}{\partial z}-i \sum_{k \geq 2} \frac{i^{k} \beta_{k}}{k !} \frac{\partial^{k} A}{\partial t^{k}}=i \gamma\left(1+\frac{i}{\omega_{0}} \frac{\partial}{\partial t}\right) \\
\times\left[A(z, t) \int_{-\infty}^{t} R\left(t^{\prime}\right)\left|A\left(z, t-t^{\prime}\right)\right|^{2} d t^{\prime}\right] .
\end{aligned}
$$

Here, $A=A(z, t)$ is the electric field envelope, the $\beta_{k}$ 's are the usual dispersion coefficients at center frequency $\omega_{0}$, and $\gamma=n_{2} \omega_{0} /\left(c A_{\text {eff }}\right)$ is the nonlinear coefficient, with $n_{2} \simeq 3.0 \times$ $10^{-20} \mathrm{~m}^{2} / \mathrm{W}$ the nonlinear refractive index and $A_{\text {eff }}$ the fiber effective area. The right hand side of (1) models self-phase modulation (SPM), self-steepening, optical shock formation, and intrapulse Raman scattering. The response function $R(t)=$ $\left(1-f_{R}\right) \delta(t)+f_{R} h_{R}(t)$ includes both instantaneous electronic and delayed Raman contributions, with $f_{R}=0.18$ representing the contribution of the Raman response to the instantaneous nonlinear polarization. For $h_{R}$, we used the experimentally determined Raman response of silica fibers. Fiber loss was neglected. The presence of quantum noise was taken into account phenomenologically by including in the input field a noise seed of one photon per mode with random phase [30].

The fiber parameters were based on a $10-\mathrm{cm}$ pure fused silica strand of diameter $2.5 \mu \mathrm{m}$ surrounded by air. This is similar to the tapered fiber described in [15], in which SC spectra with more than an octave of bandwidth were generated. The fiber dispersion for the fundamental $\mathrm{HE}_{11}$ mode was calculated using standard techniques [31], and for comparison with previously published dispersion data in PCF and tapered fiber [15], [32], Fig. 1 plots the calculated GVD in terms of both the dispersion parameter $D$ (in ps $/ \mathrm{nm} / \mathrm{km}$ ) and $\beta_{2}$ (in $\mathrm{ps}^{2} / \mathrm{m}$ ). Since both the dispersion and nonlinearity characteristics of this strand (in particular a ZDW at $780 \mathrm{~nm}$ ) are nearly identical to those of a large air-fill fraction PCF [15], the simulation results presented below can be considered generally applicable both to tapered fibers and PCF.

The effect of dispersion in the simulations was found to be accurately modelled over the range $300-1600 \mathrm{~nm}$ using terms up to $\beta_{7}$ in the Taylor series expansion, with higher order terms having negligible influence. The particular values at $850 \mathrm{~nm}$ were $\beta_{2}=-1.276 \times 10^{-2} \mathrm{ps}^{2} \cdot \mathrm{m}^{-1}, \beta_{3}=8.119 \times 10^{-5}$ $\mathrm{ps}^{3} \cdot \mathrm{m}^{-1}, \beta_{4}=-1.321 \times 10^{-7} \mathrm{ps}^{4} \cdot \mathrm{m}^{-1}, \beta_{5}=3.032 \times 10^{-10}$ $\mathrm{ps}^{5} \cdot \mathrm{m}^{-1}, \beta_{6}=-4.196 \times 10^{-13} \mathrm{ps}^{6} \cdot \mathrm{m}^{-1}, \beta_{7}=2.570 \times 10^{-16}$ $\mathrm{ps}^{6} \cdot \mathrm{m}^{-1}$. The integration of the propagation equation was carried out using the enhanced split-step-Fourier scheme described in [29], and typically required at least $2^{12}$ time and frequency discretization points and a longitudinal step size $<10 \mu \mathrm{m}$ in order to avoid numerical instabilities [30], [33].

With these fiber parameters, the numerical simulations based on (1) will be seen to adequately model the experimentally re-

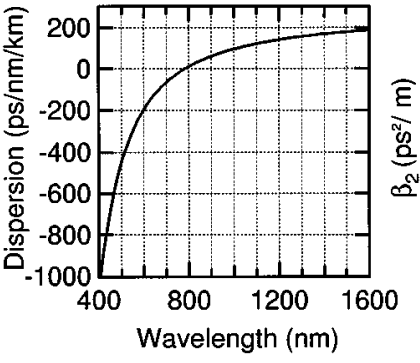

(a)

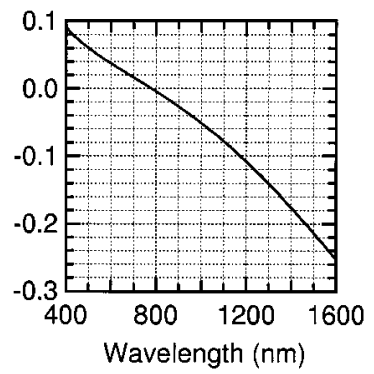

(b)

Fig. 1. Calculated GVD characteristics for the fused silica fiber strand used in numerical simulations.

ported SC structure and bandwidth for 50-150-fs input pulses. The use of shorter pulses or longer fiber lengths, however, may require effects such as fiber loss, polarization coupling [34], and the wavelength dependence of the effective area [35] to be included. For some input pulse and fiber parameter ranges, it may even be necessary to use alternative numerical methods based on the direct solution of Maxwell's equations [19].

\section{Simulation RESUlTS}

\section{A. Results in the Absence of Input Pulse Noise}

Simulations were first carried out in the absence of noise on the input pulse, using hyperbolic secant input pulses with a peak power of $10 \mathrm{~kW}$ and a wavelength of $850 \mathrm{~nm}$ in the fiber anomalous dispersion regime. These input pulse parameters are typical of those used in experimental studies [15]. Fig. 2 shows simulation results for input pulse durations (FWHM) of (a) $50 \mathrm{fs}$ and (b) $150 \mathrm{fs}$, plotting the spectral and temporal evolution at different points along the fiber.

Although the higher nonlinearity associated with PCF and tapered fibers leads to enhanced signatures of nonlinear and dispersive interactions during propagation, we stress that ultrashort pulse propagation in optical fibers has been the subject of extensive research for over 20 years [36], and many of the observed spectral and temporal features seen in the simulation results in Fig. 2 (and in experiments) can be readily interpreted in terms of well-understood physics [30], [37]. In particular, the dramatic spectral broadening observed during the SC generation process arises from the interaction between the fiber dispersion and various $\chi^{(3)}$ nonlinear processes, including SPM, four-wave mixing (FWM), self-steepening, and stimulated Raman scattering [30]. For pulses injected in the fiber anomalous dispersion regime (such as in our simulations), the initial stage of propagation is associated with strong temporal compression and approx- 

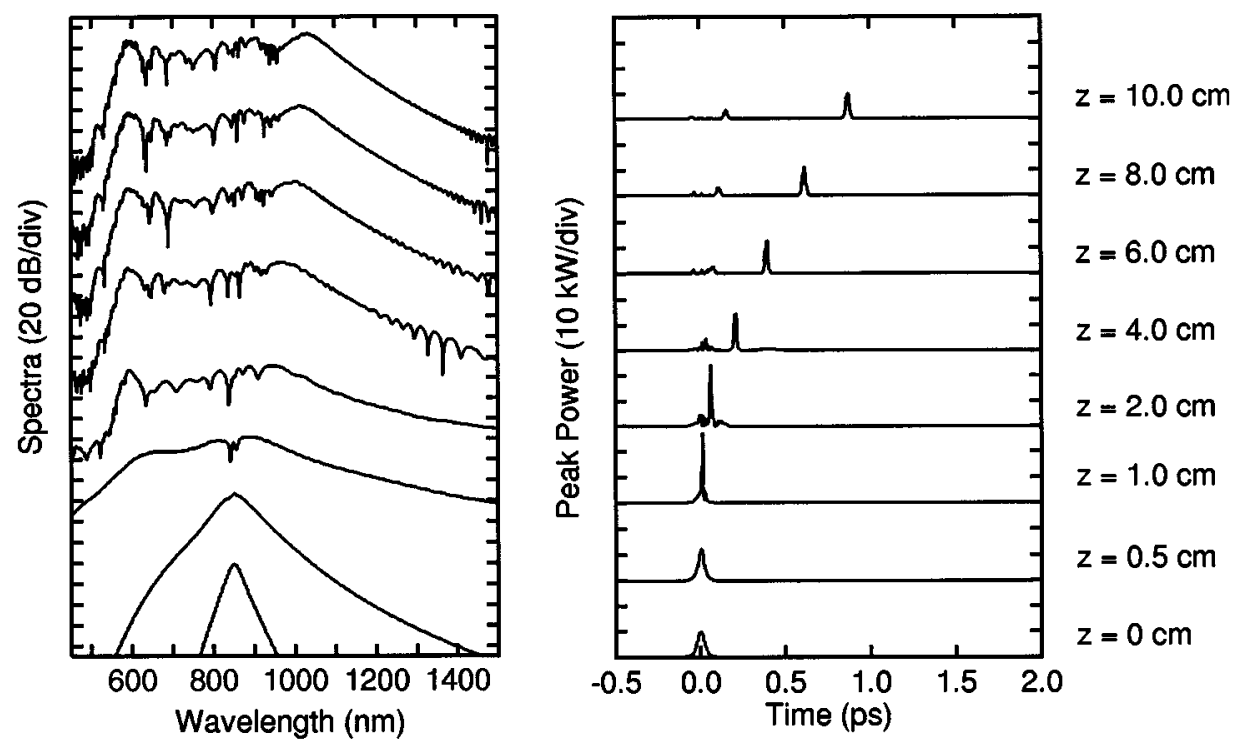

(a)
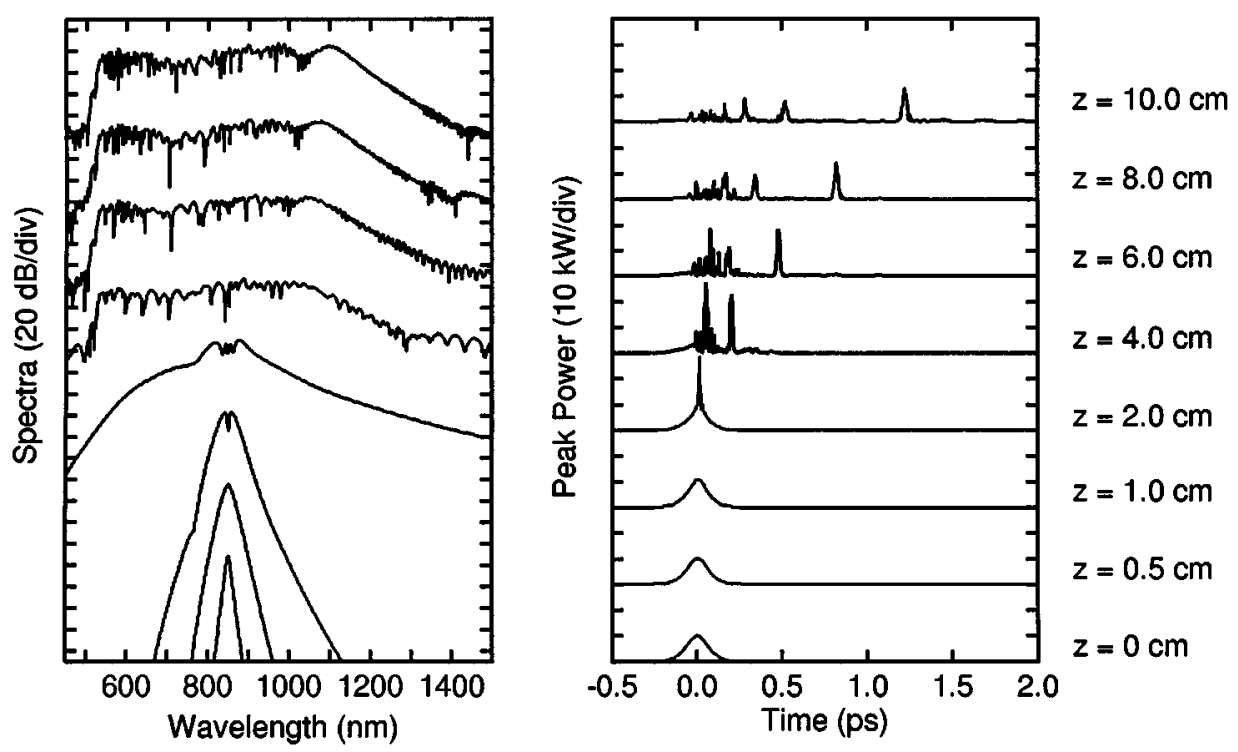

(b)

Fig. 2. Evolution of (a) 50- and (b) 150 -fs input pulses along $10 \mathrm{~cm}$ of fiber, showing both the spectral (left) and temporal (right) properties.

imately symmetrical spectral broadening, followed by more severe pulse breakup due to the development of ultrafast temporal oscillations on the injected pulse envelope from modulational instability (MI)-like effects. These processes are largely determined by the interaction of SPM and GVD, although higher order dispersion plays an increasingly important role as new spectral components are generated in the vicinity of the fiber ZDW and introduces some spectral asymmetry.

Referring back to Fig. 2, the onset of the pulse breakup can be seen to occur after $\simeq 2 \mathrm{~cm}$ for the 50 -fs input pulse and after $\simeq 4$ $\mathrm{cm}$ for the $150-\mathrm{fs}$ pulse. The fact that the 50 -fs input pulse experiences significantly more spectral broadening and compression in the first few centimeters of propagation is consistent with the increased effect of SPM on shorter pulses. The spectral broadening continues with further propagation and, quantifying the spectral width at the 20-dB point as in [15], we find that both output spectra in Fig. 2 extend over an octave from 550-1100 $\mathrm{nm}$. However, although the output spectra are essentially continuous over this range, the spectral intensity does exhibit a somewhat complex structure, with particularly distinct peaks on the long wavelength side of the spectrum in the anomalous dispersion regime. Such structure has been previously reported in experiments, for example Fig. 5(b) of [10], Fig. 4 of [13] and Fig. 6 of [15]. In the time domain, the development of this spectral structure is seen to be associated with the formation of a series of distinct high intensity pulses on the trailing edge of the residual input pulse.

As discussed by Islam et al. in [21], these distinct pulses are Raman solitons whose generation is seeded from the MI-induced oscillations developed on the propagating pulse envelope. As they propagate in the fiber, they undergo a continuous shift to longer wavelengths because of the soliton self-frequency shift, 

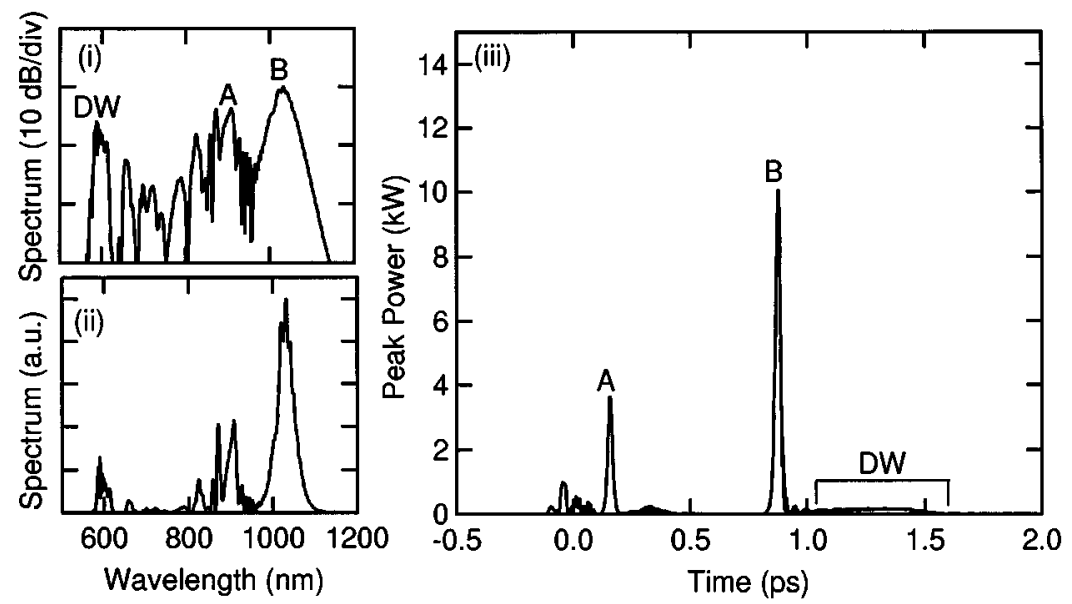

(a)
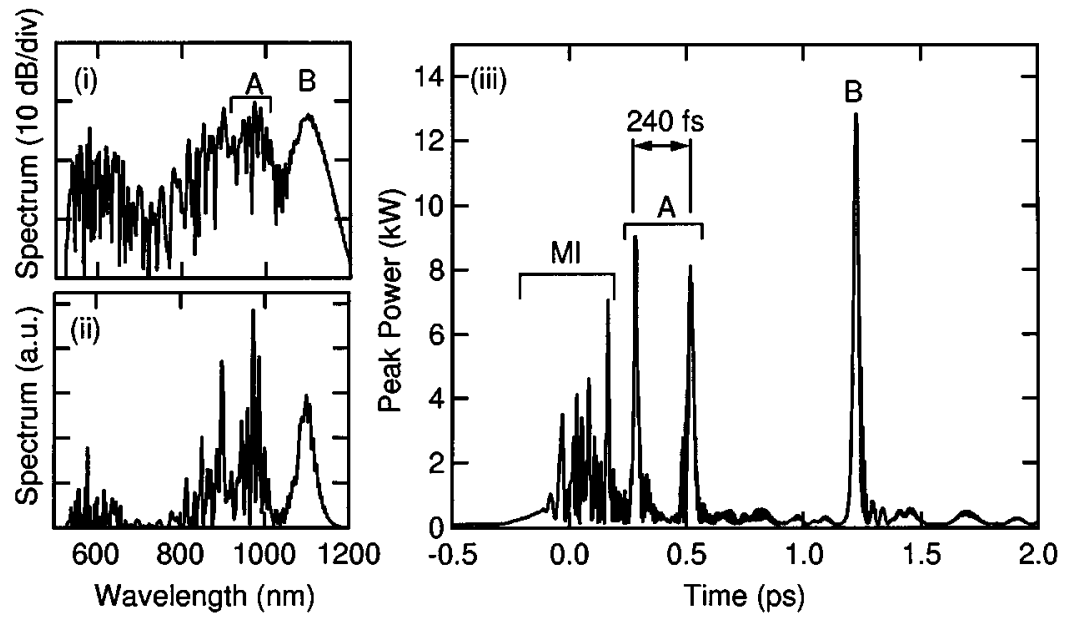

(b)

Fig. 3. Detail of output characteristics for (a) 50- and (b) 150-fs input pulses. (i) and (ii) show the spectra on logarithmic and linear scales respectively, while (iii) shows the temporal intensity. The various labeled features are discussed in the text.

and walk off temporally from the pump. Fig. 3 shows in detail the output spectral and temporal profiles to illustrate more clearly the relationship between the spectral and temporal features of these Raman solitons. In particular, numerical filtering allows the anomalous dispersion regime spectral peaks to be associated with the corresponding time-domain Raman solitons, shown as the labeled features A and B on the figure. In fact, we note that for the 150 -fs case, the time-domain manifestation of the spectral feature A (between $920-1050 \mathrm{~nm}$ ) actually corresponds to two Raman solitons separated by $240 \mathrm{fs}$; the corresponding spectral peaks partially overlap and thus exhibit a $13-\mathrm{nm}(\simeq 4.2 \mathrm{THz})$ spectral modulation.

The figure shows some additional interesting features. For the 150-fs case, the strong temporal oscillations due to MI are identified on the envelope of the residual input pulse, and for the 50-fs case, we have also identified a normal dispersion regime peak around $600 \mathrm{~nm}$ (labeled DW), which arises from the transfer of energy from the anomalous dispersion regime via the generation of a low amplitude dispersive-wave [38]. The associated time-domain manifestation of this in terms of a low amplitude broad pulse is clearly identified in the figure. Although such dispersive wave generation also occurs for the
150 -fs input pulse, the spectral and temporal signatures in this case are less distinct and therefore not specifically identified on the figure. Of course, with significant spectral content present on both sides of the fiber ZDW at $780 \mathrm{~nm}$, FWM and cross-phase modulation are also likely to play an important role in determining the overall form of the SC spectrum, but a quantitative discussion of these effects in terms of the results in Fig. 3 is not considered here.

\section{B. Results in the Presence of Input Pulse Noise}

The simulations described above for input 50- and 150-fs pulses were then repeated with the inclusion of a one photon per mode noise level on the input field. Multiple simulations were performed under identical conditions except for the initial random noise present on the input field. For the injected 50-fs pulses, no significant differences in either the spectral or temporal characteristics from run to run were observed. However, for the injected 150-fs pulses, the presence of input pulse noise led to significant run to run differences in both the output spectra and corresponding temporal profiles.

Fig. 4 shows results for five such simulations using 150-fs pulses, clearly showing run to run variations in the structure 


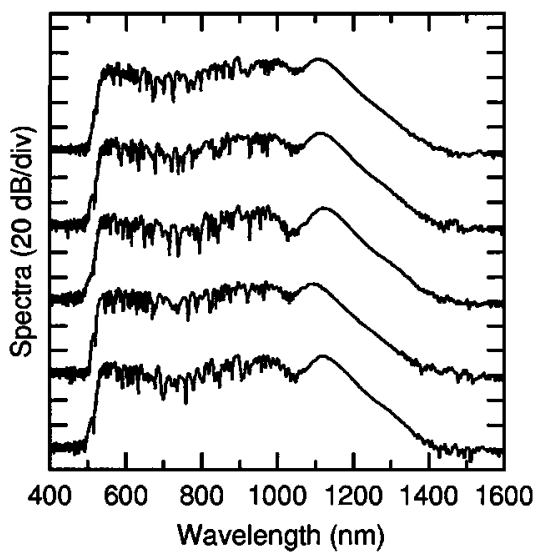

(a)

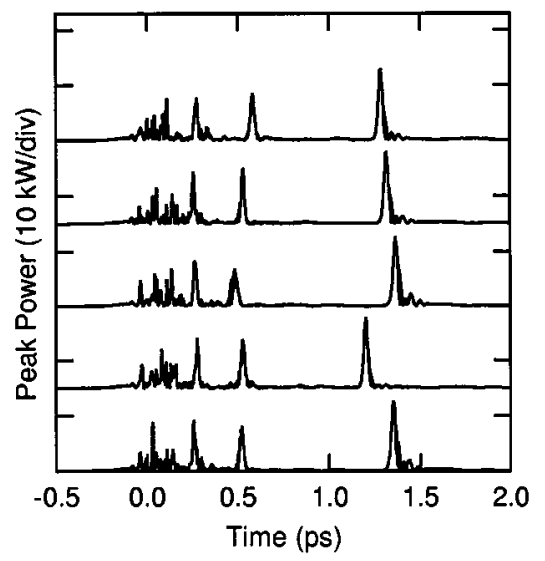

(b)

Fig. 4. Spectra (left) and temporal intensities (right) for five simulations using a $10-\mathrm{kW}$ peak power 150 -fs input pulse at a wavelength of $850 \mathrm{~nm}$.

of the observed spectra and temporal intensity. The significant jitter in the temporal position of the generated Raman solitons is particularly apparent, associated with shifts in the wavelengths of the corresponding peaks in the spectrum. These observations are consistent with previous studies of SC generation in conventional fibers, which have shown that broad-band spectral generation seeded from $\mathrm{MI}$ is very sensitive to noise on the input pulse [21]-[26].

As well as leading to the averaging of the temporal or spectral characteristics which would be experimentally measured with integrated or multishot detectors, this jitter is also associated with coherence degradation due to severe fluctuations in the spectral phase at each wavelength. This coherence degradation can be examined directly by calculating the standard deviation of the spectral phase across the output spectrum from a large number of independent simulations. An additional measure of the coherence degradation can be obtained by using a technique recently developed in an experimental study of SC generation in bulk media [39]. This method uses a modified Young's two source experiment where the two spatially separated sources which interfere are independently generated SC which yield a polychromatic interference pattern when combined together in the far-field. The spectral resolution of this pattern reveals distinct fringes at each wavelength in the SC spectrum, with the wavelength dependence of the fringe visibility providing a rigorous measure of the local coherence properties, since it is directly related to the modulus of the complex degree of (mutual) coherence between the independent SC sources. This measure of coherence degradation is calculated numerically using the definition

$$
\left|g_{12}^{(1)}\left(\lambda, t_{1}-t_{2}\right)\right|=\left|\frac{\left\langle E_{1}^{*}\left(\lambda, t_{1}\right) E_{2}\left(\lambda, t_{2}\right)\right\rangle}{\sqrt{\left\langle\left|E_{1}\left(\lambda, t_{1}\right)\right|^{2}\right\rangle\left\langle\left|E_{2}\left(\lambda, t_{2}\right)\right|^{2}\right\rangle}}\right| .
$$

Here, the angle brackets denote an ensemble average over independently generated pairs of SC spectra $\left[E_{1}(\lambda, t), E_{2}(\lambda, t)\right]$, and $t$ is the time measured at the scale of the temporal resolution of the spectrometer used to resolve these spectra. In practice, we have applied the ensemble average on the results of many simulations which yielded 200 pairs of SC obtained from input pulses with different random quantum noise. Also, since we are mainly interested in the wavelength dependence of the coherence, we have calculated the modulus $\left|g_{12}^{(1)}\right|$ at $t_{1}-t_{2}=0$, which would simply correspond to the fringe visibility at zero path difference in the Young's two source experiment described above. We also note that $\left|g_{12}^{(1)}\right|$ is calculated over a $\sim 250-\mathrm{GHz}$ numerical bandwidth, consistent with the spectral resolution used in typical SC experiments [9]. Additional simulations have also verified that the wavelength dependence of $\left|g_{12}^{(1)}\right|$ (which is discussed in detail below) is independent of the particular simulation resolution used.

Fig. 5 shows the results of this analysis applied to the 150-fs results discussed above. Fig. 5(a) and (d) show, respectively, the mean spectrum and temporal intensity profile calculated over the ensemble of simulations. It is clear that there is significant averaging of the fine structure which was previously apparent in Fig. 3(b). (Interestingly, it was noted as early as 1989 that, even though the individual spectra may possess considerable structure, the averaging over Raman soliton pulses frequency shifted by different amounts because of noise can lead to an integrated flat smooth spectrum [21].)

In terms of the coherence degradation across the spectrum, Fig. 5(b) shows the calculated spectral phase deviation $(\sigma / 2 \pi)$ at each wavelength in the spectrum. Although there is a slight decrease in $(\sigma / 2 \pi)$ around $850 \mathrm{~nm}$, the wavelength dependence of the phase stability is not clearly manifested in this figure. As expected, however, variations in the phase stability are more strongly manifested in the degree of mutual coherence $\left|g_{12}^{(1)}\right|$ plotted in Fig. 5(c), and therefore it is this which we use in the remainder of the paper to discuss the coherence degradation in more detail.

In particular, to study how the decoherence is correlated with the evolution of the injected input pulse, we have plotted in Fig. 6 typical temporal and spectral characteristics at different propagation distances. The top curves show the temporal intensity distribution obtained from one particular simulation (single shot), while the bottom curves show the spectral characteristics calculated from the ensemble average. Here, we plot both the mean spectrum (left axis) and $\left|g_{12}^{(1)}\right|$ (right axis). During the initial stage of evolution at $z=2 \mathrm{~cm}$ when the input $150-\mathrm{fs}$ pulse undergoes significant compression and associated spectral 


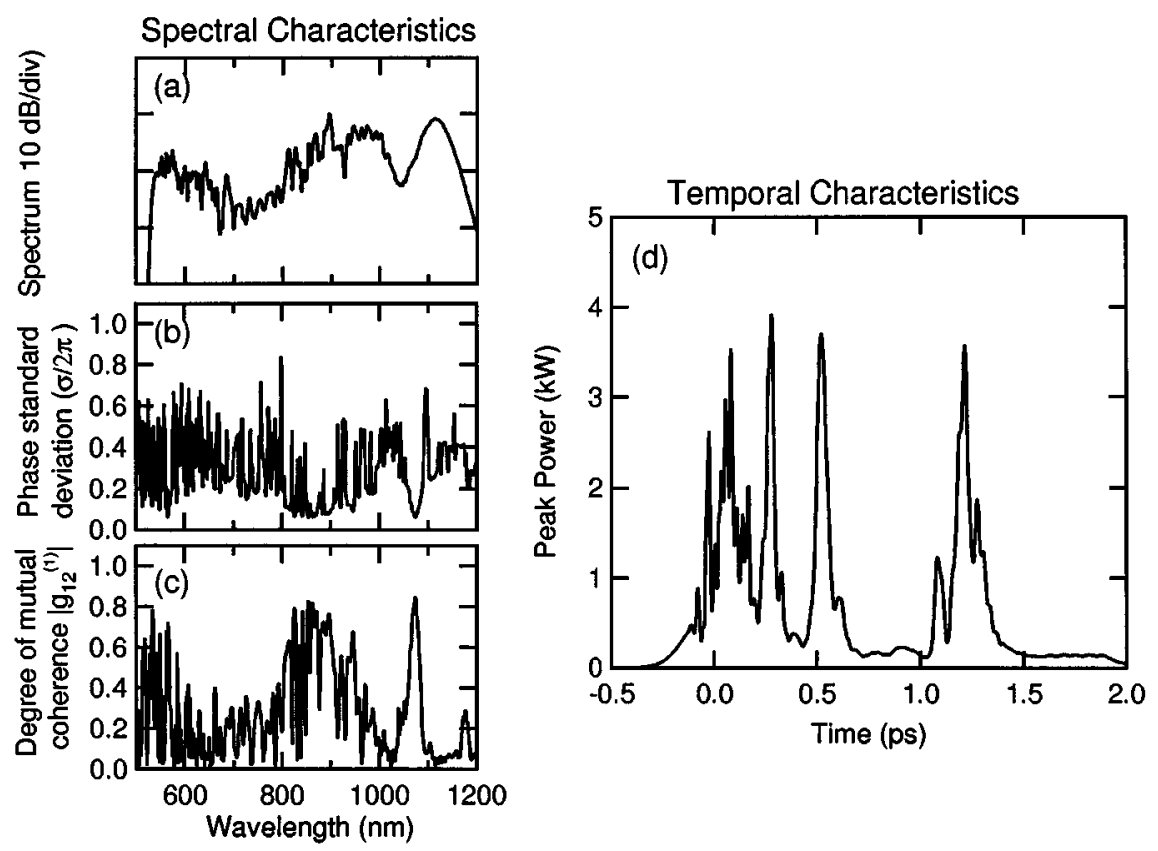

Fig. 5. Averaged output characteristics for multiple simulations of an injected 150 -fs pulse in the presence of a random one photon per mode noise level. The spectral characteristics show (a) the calculated mean spectrum, (b) the standard deviation of the spectral phase at each wavelength in the spectrum (in units of $2 \pi$ ), and (c) the corresponding degree of mutual coherence (defined in the text). The temporal characteristics in (d) show the calculated mean intensity profile.

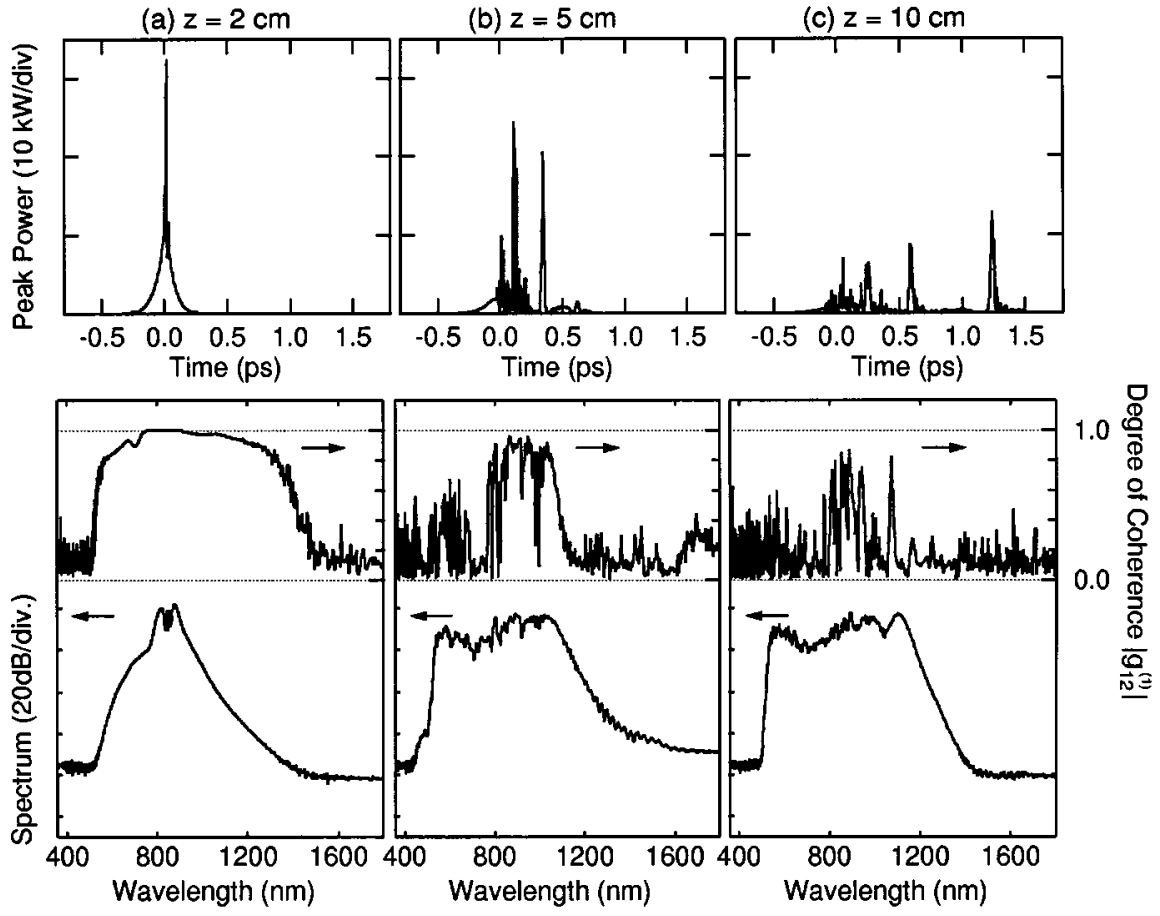

Fig. 6. For propagation distances of (a) $2 \mathrm{~cm}$, (b) $5 \mathrm{~cm}$, and (c) $10 \mathrm{~cm}$, the top curves show the temporal intensity distribution obtained from one simulation, while the bottom curves show the mean spectrum (left axis) and degree of coherence (right axis) calculated from an ensemble average.

broadening, $\left|g_{12}^{(1)}\right| \approx 1$ over the major part of the SC. However, with an increased propagation distance of $z=5 \mathrm{~cm}$ where the effects of MI and pulse breakup are clearly manifested on the temporal intensity profile, there is significant coherence degradation, with a reduced $\left|g_{12}^{(1)}\right|$. The increased spectral broadening with further propagation is associated with continued coherence degradation so that $\left|g_{12}^{(1)}\right| \ll 1$ over most of the SC spectrum at the fiber output for $z=10 \mathrm{~cm}$.
Significantly, setting the Raman gain to zero and repeating these simulations yielded similar coherence properties, implying that it is the sensitivity of MI to input pulse noise and not any effect due to stimulated Raman scattering which is the primary cause of the coherence degradation under our conditions. This suggests that superior coherence properties would be expected for SC generated using shorter input pulses where SPM plays a more significant role in the spectral broadening, 
(a) 150 fs input

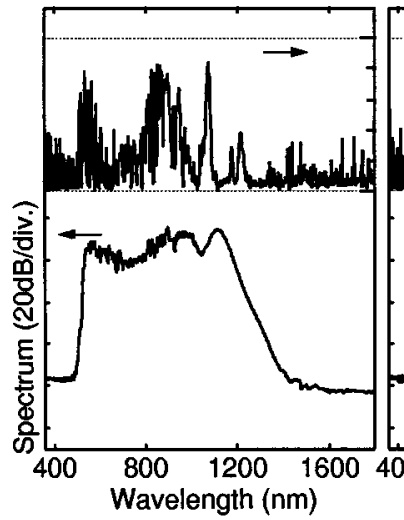

(b) 100 fs input

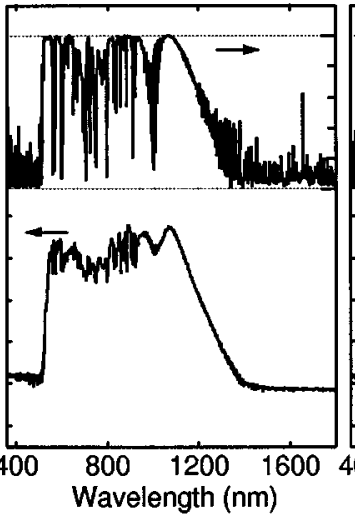

(c) 50 fs input

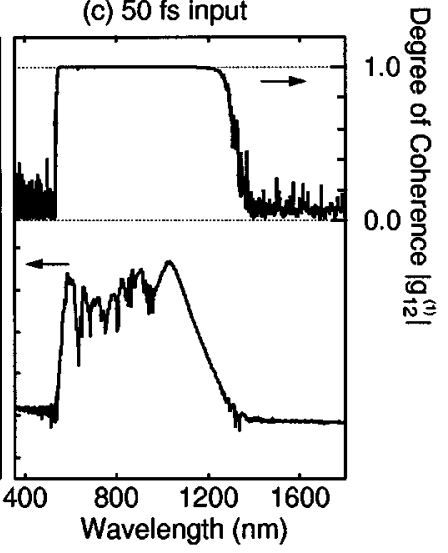

Fig. 7. Mean spectrum (left axis) and calculated degree of coherence (right axis) obtained with $10-\mathrm{kW}$ pulses at $850 \mathrm{~nm}$ for the different input pulse durations shown.

(a) $\lambda=740 \mathrm{~nm}$

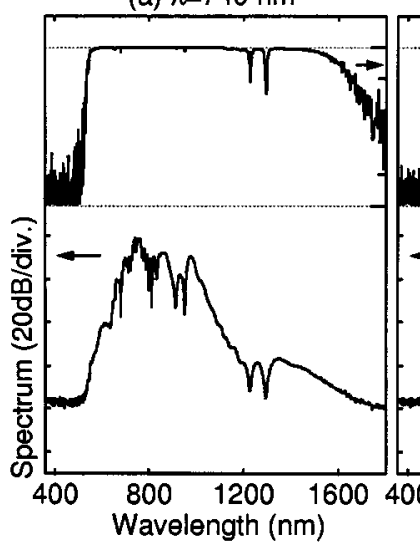

(b) $\lambda=780 \mathrm{~nm}$

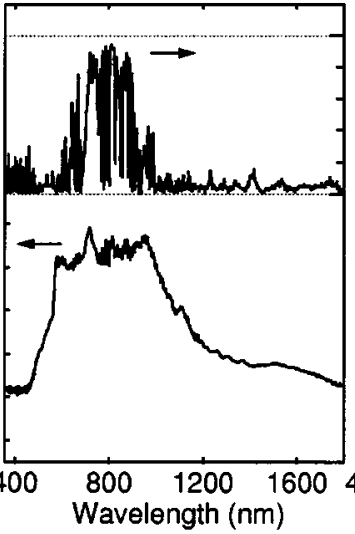

(c) $\lambda=820 \mathrm{~nm}$

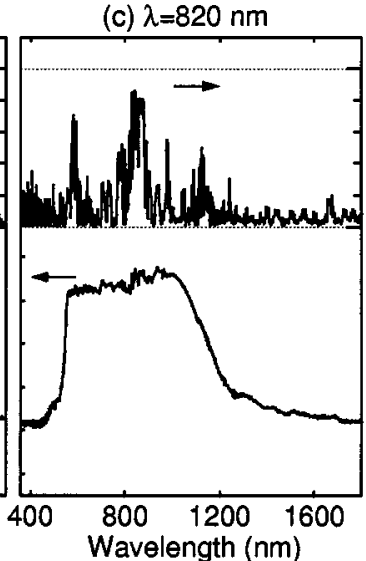

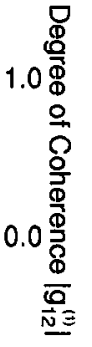

Fig. 8. Mean spectrum (left axis) and calculated degree of coherence (right axis) obtained with 10-kW 150-fs duration pulses for the different input pulse wavelengths shown.

and the effects of MI are reduced. Indeed, this is consistent with our observation that there was negligible run to run jitter with 50-fs input pulses, and confirmed more quantitatively in Fig. 7, which compares the simulation results for 150-fs input pulses with those obtained with shorter input pulse durations of 100 and $50 \mathrm{fs}$ (the wavelength and peak power were maintained at $850 \mathrm{~nm}$ and $10 \mathrm{~kW}$, respectively). It is clear that, although the spectral broadening in all cases is comparable, the coherence properties improve significantly as the input pulse duration is decreased and, indeed $\left|g_{12}^{(1)}\right| \approx 1$ over more than an octave for the shortest input pulses of $50 \mathrm{fs}$.

Additional confirmation of the influence of MI on the coherence degradation is presented in Fig. 8, which shows the results of simulations using $10-\mathrm{kW}$ peak power 150 -fs input pulses injected over a range of different wavelengths. With an input wavelength of $740 \mathrm{~nm}$ in the normal dispersion regime where $\mathrm{MI}$ is completely inhibited, there is negligible coherence degradation. The generated spectral width of $350 \mathrm{~nm}$ at the $20-\mathrm{dB}$ level is, however, significantly less than that obtained with an anomalous dispersion regime pump. The significant coherence degradation observed at $780 \mathrm{~nm}$ (the ZDW) and $820 \mathrm{~nm}$ (anomalous dispersion) is consistent with the increased influence of MI at these longer wavelengths.

\section{DiscUSSION AND CONCLUSION}

The results presented here have several important implications. Firstly, it is clear that the bandwidth and apparent uniformity of a generated SC spectrum are in themselves insufficient measures of its suitability for applications in frequency metrology. Because of the sensitivity of the spectral broadening mechanisms to noise, spectral uniformity can arise as a result of averaging effects, and broad bandwidth SC can, in fact, be associated with large spectral phase fluctuations. With the power and fiber parameters used in our simulations, the results suggest that suitable sources for metrology spanning over an octave in bandwidth and possessing excellent coherence properties require input pulses of duration 50 fs or less. Indeed, we note that this is the pulse duration range which has been used in successful PCF-based metrology experiments to date [20]. We also note that the results presented here illustrate the importance of using stabilized pump lasers in order to obtain the best coherence properties.

In addition, the use of the complex degree of coherence provides a convenient means of quantifying the coherence degradation across SC spectra generated in PCF and tapered fibers, and we anticipate that its experimental characterization 
will be readily implemented. We also note that the loss of coherence may prove a limiting factor in experiments using multishot meaurement techniques to perform intensity and phase characterization of SC generation, and that in the presence of significant phase instability, single-shot characterization would be required.

In the particular context of SC generation in PCF, an important extension of the work described in this paper is to consider in detail how the spectral and temporal SC chararteristics vary with changes in the fiber structure. Although a complete analysis of the propagation problem is, of course, possible via the direct solution of Maxwell's equations, for many PCF structures based in a regular geometrical air-hole distribution, the fiber characteristics can be readily included in the extended NLSE model above via modified fiber dispersion and effective area parameters [40]. In the case of fiber structures associated with very complex dispersion characteristics, however, it is important to ensure that the number of terms in the Taylor series expansion is sufficiently high, so that the dispersion is modeled accurately over the wavelength range of interest.

Finally, although we have discussed some strong qualitative similarities between our simulation results and experiments, it is appropriate here to consider whether a more quantitative analysis is possible. In this regard, it is important to note that the SC generation process is highly sensitive to the input pulse characteristics, and experimental uncertainties in parameters such as the injected pulse power preclude a more quantitative comparison of our results with published SC generation results. We note, nonethless, that in a recent experiment, studying Raman soliton propagation in PCF where the injection conditions and even the presence of input pulse chirp were carefully characterized, very good quantitative agreement between the extended NLSE model and experiment was reported [41].

\section{ACKNOWLEDGMENT}

The authors would like to thank S. Ralph, B. Washburn, P. Lacourt, L. Provino, and R. Trebino for stimulating discussions.

\section{REFERENCES}

[1] J. C. Knight, T. A. Birks, P. St. J. Russell, and D. M. Atkin, "All-silica single-mode optical fiber with photonic crystal cladding," Opt. Lett., vol. 21, pp. 1547-1549, 1996.

[2] T. A. Birks, J. C. Knight, and P. St. J. Russell, "Endlessly single-mode photonic crystal fiber," Opt. Lett., vol. 22, pp. 961-963, 1997.

[3] D. Mogilevtsev, T. A. Birks, and P. St. J. Russell, "Group velocity dispersion in photonic crystal fibers," Opt. Lett., vol. 23, pp. 1662-1664, 1998.

[4] T. M. Monro, D. J. Richardson, N. G. R. Broderick, and P. J. Bennett, "Holey optical fibers: An efficient modal model," J. Lightwave Technol., vol. 17, pp. 1093-1102, 1999.

[5] A. Ferrando, E. Silvestre, J. J. Miret, and P. Andrés, "Nearly zero ultraflattened dispersion in photonic crystal fibers," Opt. Lett., vol. 25, pp. 790-792, 2000.

[6] J. C. Knight, J. Arriaga, T. A. Birks, A. Ortigosa-Blanch, W. J. Wadsworth, and P. St. J. Russell, "Anomalous dispersion in photonic crystal fiber," IEEE Photon. Technol. Lett., vol. 12, pp. 807-809, July 2000 .
[7] N. G. R. Broderick, T. M. Monro, P. J. Bennett, and D. J. Richardson, "Nonlinearity in holey optical fibers: Measurement and future opportunities," Opt. Lett., vol. 24, pp. 1395-1397, 1999.

[8] W. J. Wadsworth, J. C. Knight, A. Ortigosa-Blanch, J. Arriaga, E. Silvestre, and P. St. J. Russell, "Soliton effects in photonic crystal fibers at 850 nm," Electron. Lett., vol. 36, pp. 53-55, 2000.

[9] J. K. Ranka, R. S. Windeler, and A. J. Stentz, "Visible continuum generation in air-silica microstructure optical fibers with anomalous dispersion at $800 \mathrm{~nm}$, , Opt. Lett., vol. 25, pp. 25-27, 2000.

[10] J. K. Ranka and R. S. Windeler, "Nonlinear interactions in air-silica microstructure optical fibers," Opt. Photon. News, vol. 11, no. 8, pp. 20-25, 2000

[11] I. Hartl, X. D. Li, C. Chudoba, R. K. Ghanta, T. H. Ko, J. G. Fujimoto, J. K. Ranka, and R. S. Windeler, "Ultrahigh resolution optical coherence tomography using continuum generation in an air-silica microstructure optical fiber," Opt. Lett., vol. 26, pp. 608-610, 2001.

[12] D. J. Jones, S. A. Diddams, J. K. Ranka, A. Stentz, R. S. Windeler, J. L. Hall, and S. T. Cundiff, "Carrier-envelope phase control of femtosecond mode-locked lasers and direct optical frequency synthesis," Science, vol. 288, pp. 635-639, 2000.

[13] S. A. Diddams, D. J. Jones, J. Ye, T. M. Fortier, R. S. Windeler, S. T. Cundiff, T. W. Hänsch, and J. L. Hall, "Toward the ultimate control of light: Optical frequency metrology and the phase control of femtosecond pulses," Opt. Photon. News, vol. 11, no. 10, pp. 16-22, 2000.

[14] S. A. Diddams, D. J. Jones, J. Ye, S. T. Cundiff, J. L. Hall, J. K. Ranka, R. S. Windeler, R. Holzwart, T. Udem, and T. W. Hänsch, "Direct link between microwave and optical frequencies with a $300 \mathrm{THz}$ femtosecond laser comb," Phys. Rev. Lett., vol. 84, pp. 5102-5105, 2000.

[15] T. A. Birks, W. J. Wadsworth, and P. St. J. Russell, "Supercontinuum generation in tapered fibers," Opt. Lett., vol. 25, pp. 1415-1417, 2000.

[16] T. P. M. Man, T. A. Birks, W. J. Wadsworth, and P. St. J. Russell, "Fabrication of indefinitely long tapered fibers for supercontinuum generation," in OSA Tech. Dig., Nonlinear Guided Waves and Their Applications . Washington, DC, 2001, Paper WB4, pp. 438-440.

[17] A. L. Gaeta, "Supercontinuum generation in microstructured fibers," in OSA Technical Dig. Conf. Lasers and Electro-Optics (CLEO 2001) . Washington, DC, 2001, Paper CMK3, p. 48.

[18] S. Coen, A. H. L. Chau, R. Leonhardt, J. D. Harvey, J. C. Knight, W. J. Wadsworth, and P. St. J. Russell, "White light supercontinuum generation with 60-ps pump pulses in a photonic crystal fiber," Opt. Lett., vol. 26, pp. 1356-1358, 2001.

[19] A. V. Husakou and J. Herrmann, "Supercontinuum generation of higherorder solitons by fission in photonic crystal fibers," Phys. Rev. Lett., vol. $87,2001$.

[20] R. Holzwarth, M. Zimmermann, T. Udem, T. W. Hänsch, P. Russbüldt, K. Gäbel, R. Poprawe, J. C. Knight, W. J. Wadsworth, and P. St. J. Russell, "White-light frequency comb generation with a diode-pumped CrLiSAF laser," Opt. Lett., vol. 26, pp. 1376-1378, 2001.

[21] M. N. Islam, G. Sucha, I. Bar-Joseph, M. Wegener, J. P. Gordon, and D. S. Chemla, "Femtosecond distributed soliton spectrum in fibers," J. Opt. Soc. Amer. B, vol. 6, pp. 1149-1166, 1989.

[22] T. Morioka, S. Kawanishi, K. Mori, and M. Saruwatari, "Transform-limited, femtosecond WDM pulse generation by spectral filtering of gigahertz supercontinuum," Electron. Lett., vol. 30, pp. 1166-1168, 1994.

[23] M. Nakazawa, K. Tamura, H. Kubota, and E. Yoshida, "Coherence degradation in the process of supercontinuum generation in an optical fiber," Opt. Fiber Technol., vol. 4, pp. 215-223, 1998.

[24] T. Morioka, K. Mori, and M. Saruwatari, "Coherent White Light Source and Optical Devices Therewith," US Patent 5923683, 1999.

[25] G. A. Nowak, J. Kim, and M. N. Islam, "Stable supercontinuum generation in short lengths of conventional dispersion-shifted fiber," Appl. Opt., vol. 38, pp. 7364-7369, 1999.

[26] H. Kubota, K. Tamura, and M. Nakazawa, "Analyses of coherence-maintained ultrashort optical pulse trains and supercontinuum generation in the presence of soliton-amplified spontaneous emission interaction," J. Opt. Soc. Amer. B, vol. 16, pp. 2223-2232, 1999.

[27] Ö. Boyraz, J. Kim, M. N. Islam, F. Coppinger, and B. Jalali, "10 Gb/s multiple wavelength coherent short pulse source based on spectral carving of supercontinuum generated in fibers," J. Lightwave Technol., vol. 18, pp. 2167-2175, 2000.

[28] K. R. Tamura, H. Kubota, and M. Nakazawa, "Fundamentals of stable continuum generation at high repetition rates," IEEE J. Quantum Electron., vol. 36, pp. 773-779, July 2000.

[29] K. J. Blow and D. Wood, "Theoretical description of transient stimulated Raman scattering in optical fibers," IEEE J. Quantum Electron., vol. 25, pp. 2665-2673, Dec. 1989. 
[30] G. P. Agrawal, Nonlinear Fiber Optics, 3rd ed. San Diego, CA: Academic, 2001.

[31] A. W. Snyder and J. D. Love, Optical Waveguide Theory: Chapman and Hall, 1983.

[32] J. K. Ranka, R. S. Windeler, and A. J. Stentz, "Optical properties of highdelta air-silica microstructure fibers," Opt. Lett., vol. 25, pp. 796-798, 2000.

[33] F. Matera, A. Mecozzi, M. Romagnoli, and M. Settembre, "Sideband instability induced by periodic power variation in long-distance fiber links," Opt. Lett., vol. 18, pp. 1499-1501, 1993.

[34] E. A. Golovchenko and A. N. Pilipetskii, "Unified analysis of four photon mixing, modulational instability and stimulated Raman scattering under various polarization conditions in fibers," J. Opt. Soc. Amer. B, vol. 11, pp. 92-101, 1994.

[35] S. V. Chernikov and P. V. Mamyshev, "Femtosecond soliton propagation in fibers with slowly decreasing dispersion," J. Opt. Soc. Amer. B, vol. 8, pp. 1633-1641, 1991.

[36] H. Nakatsuka, D. Grischkowsky, and A. C. Balant, "Nonlinear picosecond pulse propagation through optical fibers with positive group velocity dispersion," Phys. Rev. Lett., vol. 47, pp. 910-913, 1981.

[37] R. R. Alfano, Ed., The Supercontinuum Laser Source. New York: Springer-Verlag, 1989.

[38] P. K. A. Wai, C. R. Menyuk, Y. C. Lee, and H. H. Chen, "Nonlinear pulse propagation in the neighborhood of the zero-dispersion wavelength of monomode optical fibers," Opt. Lett., vol. 11, pp. 464-466, 1986.

[39] M. Bellini and T. W. Hänsch, "Phase-locked white-light continuum pulses: Toward a universal optical frequency-comb synthesizer," Opt. Lett., vol. 25, pp. 1049-1051, 2000.

[40] N. A. Mortensen, "Effective area of photonic crystal fibers," Opt. Exp., vol. 10, pp. 341-348, Apr. 2002.

[41] B. R. Washburn, S. E. Ralph, P. A. Lacourt, J. M. Dudley, W. T. Rhodes, R. S. Windeler, and S. Coen, "Tunable near-infared femtosecond soliton generation in photonic crystal fibers," Electron. Lett., vol. 37, pp. 1510-1512, Dec. 2001. 